\title{
Einsatz von Medizintechnik und Technisierung der Medizin - Reflexionen im Vorfeld der Jahrestagung der AEM 2014
}

\author{
Heiner Fangerau • Gisela Badura-Lotter
}

Online publiziert: 22. Juli 2014

(C) Springer-Verlag Berlin Heidelberg 2014

Im Jahr 1962 titelte das Nachrichtenmagazin der Spiegel „Krankenpflege: Puls am Telephon“ und stellte dem geneigten Leser leicht tendenziös „ein neuartiges Hospital-Zubehör“ vor, das Wissenschaftler des ,größten Herstellers von Fernlenkgeschossen außerhalb der USA und der Sowjet-Union“ auf den medizinischen Markt zu bringen gedachten: eine „elektronische Krankenschwester" ([1], S. 91). Es handelte sich hierbei um ein technisches System, mit dessen Hilfe auf Überwachungsstationen Vitalfunktionen von Kranken gemessen und an ein Kontrollpult übermittelt werden könnten, das wiederum von einer menschlichen Krankenschwester bedient werden sollte. Während auf der einen Seite diese Entwicklung als Lösung für verschiedene praktische Probleme angesehen wurde (z. B. Krankenschwestermangel, Hilfe bei der Erledigung von Routineaufgaben), wurden auf der anderen Seite Bedenken laut - diese reichten von der Sorge um Arbeitsplätze bis hin zur „Befürchtung, die Mechanisierung der Krankenbetreuung könne bei Patienten den Eindruck erwecken, man kümmere sich nicht um sie" ([1], S. 92).

Heute ist die hier aufscheinende Vision des Telemonitoring von Patientinnen und Patienten nicht nur auf Intensivstationen eine Realität. Auch für unterwegs und zu Hause existieren Messsysteme, die Vitalfunktionen, wie zum Beispiel den Blutdruck bestimmen, aufzeichnen und damit Technik sowohl diagnostisch als auch therapeutisch wirksam werden lassen $[6,10]$. Die Bedenken, die der Technik jedoch nach wie vor entgegengebracht werden, haben sich im Vergleich zu 1962 kaum verändert. Immer noch steht die Debatte im Raum, ob die Medizin durch zu viel Technik an Menschlichkeit verliere. Stellvertretend für diese Sorge steht das zum Schlagwort gewordene Prinzip „High person - low technology“, das die Palliativmedizin in der Mitte der 1970er Jahre hervorbrachte [7].

Prof. Dr. H. Fangerau $(\bowtie) \cdot$ Dr. G. Badura-Lotter Institut für Geschichte, Theorie und Ethik der Medizin, Universität Ulm, Frauensteige 6, 89077 Ulm, Deutschland

E-Mail: heiner.fangerau@uni-ulm.de 
In der Soziologie und Philosophie ist die Technik mit ihren Segnungen und Gefahren schon lange ein Thema. Kosten und Nutzen ihres Einsatzes werden ebenso diskutiert wie ihre ständige Ausweitung, die Technisierung des Menschen und seiner Lebenswelt [3]. Die Technik wird dabei nicht mehr nur als Hilfe bei der Arbeit, Ersatz für einzelne Mängel (Arnold Gehlen) oder Optimierungswerkzeug erlebt. Vielmehr ist sie ubiquitär in Lebensprozesse kaum mehr merklich eingebunden [11]. In der Medizin ist sie sogar im wahrsten Sinne ,unter die Haut“ gegangen und zum Beispiel in der Reproduktionsmedizin gar an der Zeugung und dem Werden eines Menschen selbst beteiligt. Diese Macht der Technik macht Angst und wird in der Literatur spätestens seit dem beginnenden 19. Jahrhundert und in den verschiedensten Spielarten der heutigen „Popkultur“ auch unter normativen Gesichtspunkten präsentiert und ausgebreitet. Solche Technikdiskurse haben die vielfältigsten Studien zum Beispiel zur Technikethik oder zur Technikfolgenabschätzung im Blick, wenn sie sich darum bemühen, die normative Kraft technischer Errungenschaften zu ermitteln oder den moralisch gebotenen Umgang mit Technik zu ergründen. Treffend stellte Christoph Hubig als einer der Philosophen, die sich der Technikethik zugewandt haben, als Diagnose der Zeit fest, dass Technikethik darauf aus sei, ,die Bedingungen einer das instrumentelle Handeln sichernden Technik selbst zu sichern“ ([8], 235 f.).

Genau an diesem Punkt beginnt auch die medizinethische Beschäftigung mit Technik. Wünschen sich Ärzte und Patienten auf der einen Seite die größtmögliche Kontrolle über ihre Physiologie, Anatomie und Psyche, um eben nicht zu erkranken, so empfinden sie doch gerade diese Kontrolle auch als Einschränkung ihrer Autonomie oder sogar als Bedrohung ihres menschlichen Selbstverständnisses. Die technisch vermittelte Kontrolle trägt hier neben dem unerwünschten Aspekt der Dehumanisierung noch den Charakter des nun in jeder Hinsicht unmenschlich potenzierten Beschränkers menschlicher Handlungsoptionen. Gleichzeitig wird gerade dieses Potential, das in der Veränderung des Menschen und seiner Lebenswirklichkeit durch die Technik liegt, durchaus auch als Vergrößerung seiner Autonomie (z. B. im Zusammenhang mit Reproduktionstechniken) oder als Erweiterung/ Verbesserung seines Soseins als Mensch (z. B. im Transhumanismus) gesehen. Im Feld der konkreten Patientenversorgung werden noch weitere normativ getränkte Dimensionen medizinischen Denkens und Handelns berührt (z. B. das Ideal der Fürsorge, Ziel der Teilhabe, Wunsch nach Sicherheit und Berechenbarkeit der Technik im Einsatz, Bedürfnis nach Wahrung der Privatheit und des Vertrauensschutzes im Umgang mit technischen Datenspeichern, Handeln in Einklang mit dem eigenen Selbstverständnis - ärztlich und von Patientenseite [9]). Daher kommt der Technik eine Rolle zu, die neben Nützlichkeitsaspekten immer auch moralische Felder und ihre theoretische Fundierung berührt. Dabei kann die Medizintechnik durch einen Zusammenstoß mit kulturellen Werten zum kritisierten Objekt werden ([5], S. 214 ff.) oder sie kann selbst auf normativer Basis zu einem moralischen Agens werden, wenn ihre bloße Existenz direkte moralische Konsequenzen zeitigt - ein Beispiel wäre die Einführung der Technik der extrakorporalen Stoßwellenlithotripsie, die mit der Forderung einhergeht, offene Steinoperationen künftig als risikoreicher und belastender für Patientinnen und Patienten zu unterlassen, auch wenn die neue Technik nicht überall vorhanden, teuer und damit ein möglicher Generator von Ungerechtigkeit ist [4].

Es existiert eine reichhaltige Literatur $\mathrm{zu}$ den hier aufscheinenden „Herausforderungen technisierter Medizin“ [2] und auch Verbindungslinien zwischen Technisierung und Medikalisierung sowie den dadurch sich zusätzlich ergebenden ethischen Konflikten in der Medizin, die u. a. in der Frage kulminieren, welcher Akteur moralisch betrachtet welchen 
Nutzen beanspruchen darf, sind bereits vielfach gezogen worden (siehe u. a. [6]). Da jedoch jede Gesellschaft, jede Generation einen neuen Umgang mit medizinischer Technik erlernt und pflegt [11] und da jede Technik ihr eigene, neue Konflikte zu evozieren scheint, ist es notwendig, sich neben den großen soziologischen und philosophischen Betrachtungslinien jeweils auch den spezifisch neuen Techniken und ihrem Einsatz in der Medizin zuzuwenden.

Die Jahrestagung der AEM 2014 hat sich zum Ziel gesetzt, beide Perspektiven, die großen Entwicklungen und die Eigenheiten der besonderen Techniken in den Blick zu nehmen. Besonders in der klinischen Medizin und in vielen Bereichen der Pflege wächst die Vielfalt hochkomplexer technischer Verfahren und Assistenzsysteme. In Diagnostik, Therapie und Prävention kommen so verschiedene Instrumente zum Einsatz wie Überwachungsgeräte für Vitalparameter, Operationsroboter, elektronische Implantate oder neurotechnische Arm- und Beinprothesen. Auf der Jahrestagung der AEM 2014 sollen ethische Fragen, die sich im Kontext einer intensiven Techniknutzung im Gesundheitssystem stellen, multiperspektivisch neu diskutiert werden. Wir hoffen damit, die vielfältigen Bedeutungen und die Tragweite der aktuellen heterogenen Medizintechniken für die Medizin (verstanden als menschlicher Handlungsraum) erfassen zu können.

\section{Literatur}

1. Anonymus (1962) Puls am Telephon. Der Spiegel 15:90-92

2. Aurenque D, Orsolya F (2014) Medizinphilosophie oder philosophische Medizin? Philosophisch-ethische Beiträge zu Herausforderungen technisierter Medizin. Frommann-Holzboog Verlag, Stuttgart

3. Blumenberg H (2009) Geistesgeschichte der Technik. Suhrkamp, Frankfurt

4. Braun H (2007) Ein neuer Markt in der Medizintechnik: Einführung und Ausbreitung der Extrakorporalen Stoßwellenlithotripsie (ESWL) im Krankenhaussektor. In: Braun H-J (Hrsg) Gesundheit durch Technik? Technik und Medizin seit dem Ende des 19. Jahrhunderts. Georg-Agricola-Ges., Freiberg, S 63-81

5. Fangerau H (2010) Spinning the Scientific Web: Jacques Loeb (1859-1924) und sein Programm einer internationalen biomedizinischen Grundlagenforschung. Akademie Verlag, Berlin

6. Fangerau H, Martin M (im Druck) Blutdruck messen: Die „Technikalisierung“ der Kreislaufdiagnostik. In: Sigelen A (Hrsg) Herzblut - Geschichte und Zukunft der Medizintechnik - Ausstellungskatalog. Technomusuem, Mannheim

7. Holden C (1980) The hospice movement and its implications. Ann Am Acad Pol Soc Sci 447:59-63

8. Hubig C (2006) Die Kunst des Möglichen. Teil 1: Technikphilosophie als Reflexion der Medialität. Transcript, Bielefeld

9. Manzeschke A, Weber K, Rother E, Fangerau H (2013) Ergebnisse einer Studie. Ethische Fragen im Bereich Altersgerechter Assistenzsysteme. Studie im Auftrag der VDI/VDE Innovation und Technik $\mathrm{GmbH}$ im Rahmen der vom Bundesministerium für Bildung und Forschung (BMBF) beauftragten Begleitforschung AAL. Ludwigsfelde

10. Middeke M (2009) Hochdruckwarnung per SMS. MMW Fortschr Med 151:47-51

11. Sackmann R, Weymann A (1994) Die Technisierung des Alltags. Generationen und technische Innovationen. Campus, Frankfurt 